\title{
Hydrostatic pressure dependence of the luminescence and Raman frequencies in polyfluorene
}

\author{
C. M. Martin, ${ }^{1}$ S. Guha, ${ }^{2, *}$ M. Chandrasekhar, ${ }^{1}$ H. R. Chandrasekhar, ${ }^{1}$ R. Guentner, ${ }^{3}$ P. Scanduicci de Freitas,${ }^{3}$ and U. Scherf ${ }^{3}$ \\ ${ }^{1}$ Department of Physics, University of Missouri, Columbia, Missouri 65211, USA \\ ${ }^{2}$ Department of Physics, Astronomy and Materials Science, Southwest Missouri State University, Springfield, Missouri 65804, USA \\ ${ }^{3}$ Bergische Universität Wuppertal, Makromolekulare Chemie, Wuppertal, Germany
}

(Received 12 December 2002; published 11 September 2003)

\begin{abstract}
We present studies of the photoluminescence (PL), absorption, and Raman scattering spectra from poly[2,7-(9,9'-bis(2-ethylhexyl))fluorene] under hydrostatic pressures of $0-100 \mathrm{kbar}$ at room temperature. The well-defined PL and associated vibronics that are observed at atmospheric pressure change dramatically around $20 \mathrm{kbar}$ in the bulk sample and at around $35 \mathrm{kbar}$ for the thin-film sample. Beyond these pressures the PL emission from the backbone is swamped by strong peaks due to aggregates and keto defects in the 2.1-2.6 $\mathrm{eV}$ region. The Raman peaks shift to higher energies and exhibit unexpected antiresonance line shapes at higher pressures, indicating a strong electron-phonon interaction.
\end{abstract}

DOI: 10.1103/PhysRevB.68.115203

PACS number(s): $61.50 . \mathrm{Ks}, 71.20 . \mathrm{Rv}, 78.30 . \mathrm{Jw}$

\section{INTRODUCTION}

Polyfluorenes (PFs) have emerged as attractive alternatives $^{1,2}$ to other conjugated polymers for display applications due to their efficient blue emission and high hole mobility at room temperature. ${ }^{3,4}$ Blue electroluminescent materials are of particular interest for organic displays since blue light can be converted into red and green quite easily by color-changing media (fluorescent dyes). ${ }^{5}$ PFs have been very efficiently utilized in organic light emitting diodes (OLEDs). ${ }^{6}$ They have been found to exhibit complex morphological behavior that has interesting implications due to their rich photophysics. The liquid crystallinity of PF derivatives with long alkyl substituents allows fabrication of electroluminescent devices with highly polarized emission ${ }^{7}$ that is of potential use for backlighting in liquid crystalline displays. ${ }^{8}$

OLEDs fabricated from the PF family of materials are known to degrade under operation. The desired blue electroluminescence from the singlet excitons changes to an undesirable 2.1-2.6 eV greenish/reddish emission. Photooxidation produces a similar broad emission band in the photoluminescence (PL) spectrum. This redshifted emission has been attributed to aggregation and/or excimer formation in the material. ${ }^{10,11}$ Recently, List et al. ${ }^{12}$ have conclusively shown that the $2.3-\mathrm{eV}$ band is related to emission from keto defect sites (9-fluorenone) in the sample. These defect sites act as guest emitters that can efficiently trap singlet excitons created on the conjugated polyfluorene backbone. Timeresolved PL measurements further show different temporal dynamics for the various peaks in the $2.1-2.6-\mathrm{eV}$ region, indicating that the origin of some of the peaks is due to on-chain emissive defects while other peaks are from aggregates and excimers. ${ }^{13,14}$

In this work we probe the optical properties of poly[2,7-(9,9'-bis(2-ethylhexyl))fluorene] (PF2/6) via PL, absorption, and Raman scattering as a function of hydrostatic pressure. Hydrostatic pressure enhances intermolecular interaction and changes the molecular geometry without producing chemical changes. An understanding of the influence of the intermolecular interactions is crucial since the photolu- minescence quantum yield (PLQY) of polymers is known to decrease in the solid state. For example, in methylated ladder-type poly para-phenylene (m-LPPP), the PLQY of solution and film are $100 \%$ and $30 \%$, respectively. ${ }^{15}$ In decyloxy PPP (DOPPP) PLQY values of $85 \%$ in solution and $35 \%$ in film are observed. ${ }^{16}$ In the solid state the electronic properties of organic materials depend significantly on the three-dimensional interactions. Recent theoretical methods of quantum-chemistry and solid-state physics in conjunction with experimental measurements have provided valuable insight into the electronic and optical properties of both isolated and interacting conjugated chains (oligomers and polymers). ${ }^{17-20}$ Hydrostatic pressure studies also allow us to probe the effects of enhanced interaction on the aggregate and defect-related emissions in these systems.

An additional interesting feature of PF2/6 is that its backbone conformation is intermediate to that of planar and nonplanar conjugated solids. It thus provides an interesting contrast to the planar polymer m-LPPP and the nonplanar oligophenyls such as para hexaphenyl (PHP). Our previous studies indicate that enhanced intermolecular interactions under the impact of pressure in conjugated solids typically produce an increased degree of conjugation, destabilization of localized states as in $\mathrm{m}$-LPPP, ${ }^{21,22}$ and changes in the ring torsional motion as in PHP. ${ }^{23}$ These changes in geometry can be deduced from their influence on electronic and vibrational spectra. PFs also show rich morphological behavior as a function of temperature: the glass-rubber transition and the liquid crystalline phase are induced at elevated temperatures. It therefore makes it an interesting system to study under high pressure since one can test whether any of the hightemperature phases can be induced by the application of pressure alone at room temperature, thus allowing the study of the corresponding changes in their electronic and vibrational properties in a different region of phase space.

\section{EXPERIMENTAL DETAILS}

\section{A. Methodology}

Pressure studies were conducted in a Merrill-Bassett-type diamond-anvil cell (DAC) with cryogenically loaded argon 


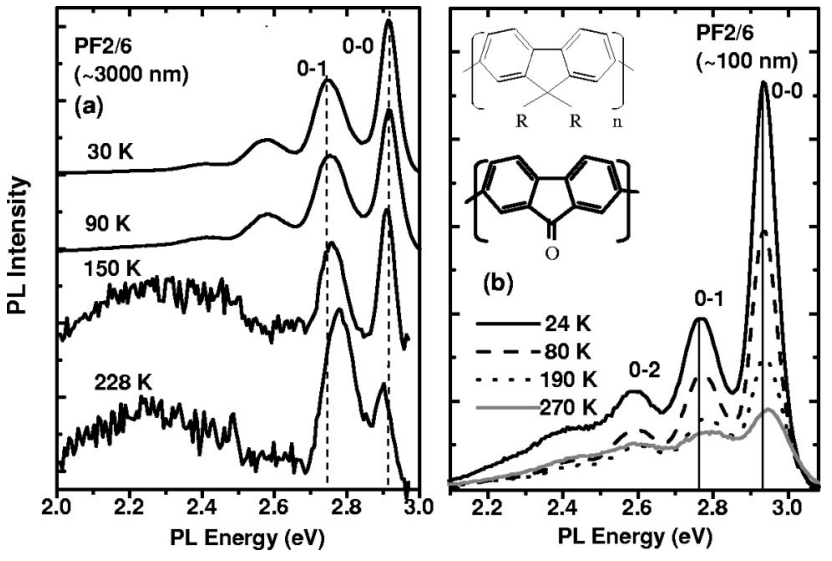

FIG. 1. PL spectrum of PF2/6 at selected values of temperature for (a) a thick and (b) a thin film. The vertical lines indicate the shift in the transition energies as temperature is increased. The top inset in (b) shows a monomer structural unit of PF2/6 where R denotes the ethyl hexyl side group; the bottom inset is the fluorenone structural unit (keto defect).

as the pressure medium. A small chip of ruby was included in the sample chamber, thus allowing the determination of pressure by measuring the shift of the luminescence spectrum of ruby. For ambient pressure measurements the samples were loaded in a cryostat that was evacuated to below 100 mTorr to prevent any photo-oxidative damage.

PL and absorption spectra were recorded using an Ocean Optics PC2000 spectrometer with $25-\mu \mathrm{m}$ slits. The 351.1-nm line from an $\mathrm{Ar}^{+}$laser was used in a backscattering configuration for PL. Raman measurements were performed using a Spex triplemate spectrometer and the 647.1-nm line of a $\mathrm{Kr}^{+}$laser with $10 \mathrm{~mW}$ incident power in a backscattering configuration. The scattered light was detected using a cryogenically cooled charge-coupled device (CCD) array detector and a holographic supernotch filter to block the elastically scattered light. Measurement of the spectrum of a chip of ruby outside the pressure chamber before each Raman measurement ensured that the calibration of the spectrometer was consistent from one day to the next. The low-temperature measurements were conducted in a closed cycle helium refrigerator. The spectral data were analyzed using PeakFit and Origin to determine the position, area, and full width at half maximum (FWHM) of the peaks.

\section{B. Sample details}

PF2/6 forms planar monomer units but has a torsional degree of freedom between adjacent monomer units shown in the inset of Fig. 1(b); its synthesis is described in Ref. 2. The torsional freedom in PF2/6 makes the structure an intermediate case between nonplanar oligophenyls and planar m-LPPP. PF2/6 is semicrystalline at room temperature, with a glass-rubber transition temperature $\left(T_{g}\right)$ of the amorphous component at $80^{\circ} \mathrm{C}$, and a crystalline to nematic liquid crystalline phase transition at $167^{\circ} \mathrm{C}$ (reverse transition at $132^{\circ} \mathrm{C}$ upon cooling). ${ }^{2}$ The molecules have been shown to have a C5 helical conformation at room temperature. ${ }^{2}$ Upon further processing some polyfluorene films display a $\beta$ phase, which has a more extended intrachain $\pi$ conjugation, in addition to the regular glassy $\alpha$ phase. The $\beta$ phase has been detected in 9,9-di-n-octyl-PF (PF8 or PFO), ${ }^{8,9}$ and shows a distinct redshift of absorption and emission peaks with a well-resolved vibronic progression both in absorption and emission. In contrast, the $\alpha$ phase shows a well-resolved vibronic progression only in the emission spectrum. Due to the branched alkyl side groups PF2/6 is not expected to easily form a $\beta$ phase, however, it has been shown that a highly aligned state can be achieved with solvent treatment and thermal cycling. ${ }^{24}$

The PL and Raman spectra were measured from a powder sample of PF2/6, which has both amorphous and crystalline components, while the absorption and PL were measured from a film. The film was prepared by drop-casting PF2/6 dissolved in spectroscopic grade dichloromethane directly onto the surface of the bottom diamond of the DAC. While the film is loosely attached to the diamond surface, it is not bonded onto it, and therefore experiences hydrostatic pressure in the DAC.

All the Raman measurements were carried out with a bulk (powder) sample. This was necessary because the Raman signal from a film is very weak due to the drastically reduced scattering volume.

\section{STEADY-STATE PHOTOLUMINESCENCE AND ABSORPTION}

\section{A. Experimental results}

Figure 1 shows the PL spectra from two PF2/6 films [panel (a) thick film, $\approx 3000 \mathrm{~nm}$; panel (b) thin film, $\approx 100 \mathrm{~nm}$ ] for a few selected values of temperature. Vibronic progressions are seen in the PL emission of both films, indicating a coupling of the backbone carbon-carbon stretch vibration to the electronic transitions. The vibronic peaks result from a nonzero overlap of different vibronic wave functions of the electronic ground and excited states. The transition highest in energy is the 0-0 transition, which takes place between the zeroth vibronic level in the excited state and the zeroth vibronic level in the ground state. The $0-1$ transition involves the creation of one phonon. The relative intensity of the $0-0$ peak to the $0-1$ peak in the thick film [Fig. 1(a)] is lower than that in the thin film [Fig. 1(b)] indicating a higher self-absorption in the thick film. The peak positions were determined by fitting the spectra to Gaussian line shapes. In the thin film, the main vibronic peaks that are observed at $30 \mathrm{~K}$ are the $0-0$ peak at $2.93 \mathrm{eV}$, the $0-1$ at 2.77 $\mathrm{eV}$, and the 0-2 transition at $2.59 \mathrm{eV}$. An additional vibronic replica is observed at $2.86 \mathrm{eV}$ between the $0-1$ and $0-2$ peaks. These values are very close to the energies observed in the thick film.

With increasing temperatures a broad peak is seen to emerge at $2.3 \mathrm{eV}$ around $150 \mathrm{~K}$ for the thick film. Recent work suggests that this peak is related to emission from keto defect sites, ${ }^{2,12}$ which are due to the inclusion of fluorenone units along the PF backbone. Keto defects [shown in the inset of Fig. 1(b)] can be accidentally incorporated into the $\pi$-conjugated PF backbone either during synthesis, by direct inclusion of a fluorenone unit, due to oxidization of nonalky- 


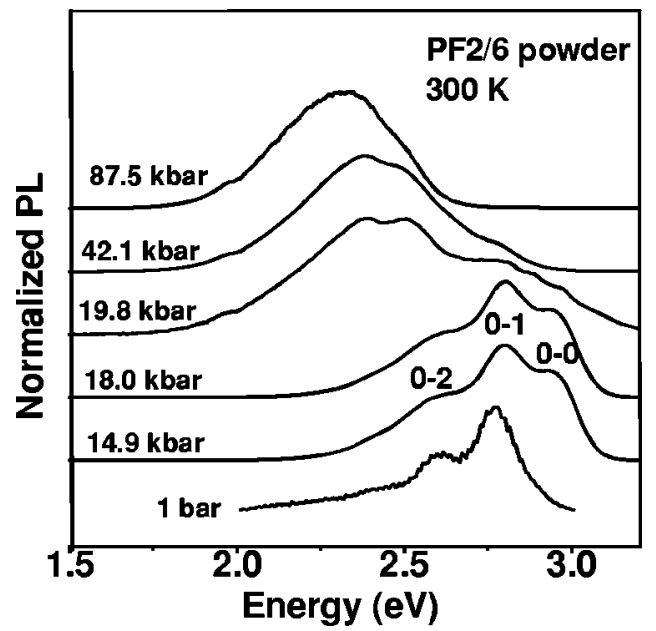

FIG. 2. PL spectrum of bulk PF2/6 at selected values of hydrostatic pressure. The 1-bar spectrum was measured from a sample evacuated to below 100 mTorr to prevent photo-oxidative damage.

lated or monosubstituted fluorene sites, or as a result of a photo-oxidative degradation process. The concentration of these defect sites is quite low in our PF2/6 sample since the $2.3 \mathrm{eV}$ emission is absent in the thin film [Fig. 1(b)]. Also, it is a thermally activated process; a weak defect-related emission is only observed for temperatures above $150 \mathrm{~K}$ for the thick film [Fig. 1(a)].

Figure 2 shows the $300-\mathrm{K}$ PL spectrum of a bulk powder sample of PF2/6 at selected values of pressure. The 0-0 transition $(2.9 \mathrm{eV})$ at ambient pressure is barely visible due to self-absorption effects. However, the other vibronics at ambient pressure correlate with the film data in Fig. 1. The most remarkable feature is the emergence of a strong orange emission above $\sim 20 \mathrm{kbar}$ at $2.4 \mathrm{eV}$, which dominates the spectrum and completely overwhelms the PL from the backbone. This peak is attributed to a combination of emissions from aggregates and keto defect sites (discussed in greater detail in Sec. III B). The broad emission, which is comprised of at least three peaks, clearly redshifts with increasing pressures. The pressure dependence of the backbone PL transitions is difficult to quantify. The apparent position of the 0-0 transition is affected by changes in the absorption spectrum, and the relative intensities of the backbone and aggregate/defect emissions change dramatically as pressure is increased. The vibronic peaks are clearly observable up to 20 kbar above which pressure they appear to remain relatively constant in energy with increasing pressure. This behavior is contrary to the observations in other $\pi$-conjugated molecules and polymers where the backbone emission clearly redshifts under increasing pressures as, for example, in polyacetylene, ${ }^{25}$ polythiophene, ${ }^{26} \mathrm{~m}$-LPPP, ${ }^{21} \mathrm{PHP}^{27}$ poly (p-phenylene vinylene) (PPV), ${ }^{28}$ and MEH-PPV. ${ }^{29}$

In order to clarify the behavior of the backbone emission which, as seen from Fig. 1, is less affected by the keto defect in thin films, we studied the pressure dependence of the PL from a film of PF2/6 that was drop-cast onto the surface of one of the diamonds of the DAC. We systematically measured the PL both for increasing and decreasing pressures. The backbone emission is clearly defined up to $30 \mathrm{kbar}$, as
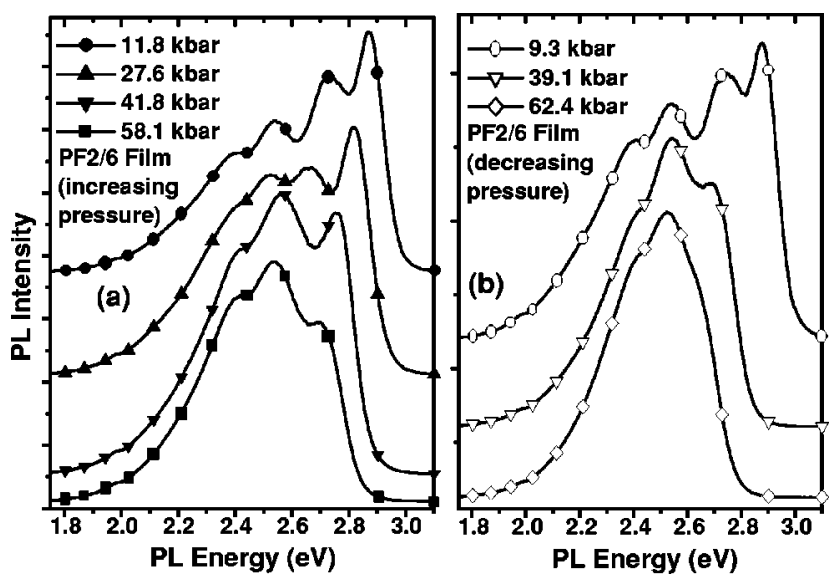

FIG. 3. Room-temperature PL spectrum of a PF2/6 film at selected values of pressure (a) while increasing the pressure and (b) on lowering the pressure.

shown in Fig. 3(a). Above 30 kbar the 0-1 and 0-2 can no longer be clearly distinguished; instead a broad peak appears at $2.5 \mathrm{eV}$ with a shoulder at $2.4 \mathrm{eV}$. The $0-0$ vibronic peak is clearly observed up to $42 \mathrm{kbar}$ as shown in Fig. 3(a). As the pressure increases further, the $2.5 \mathrm{eV}$ peak dominates the spectrum up to the highest pressures $(\sim 100 \mathrm{kbar})$. Figure 3(b) shows the PL spectra taken while lowering the pressure. Some hysteresis is observed in recovering the low-pressure spectra. In the mid-range (30-45 kbar) the emission at 2.2$2.5 \mathrm{eV}$ is still dominant and the backbone emission recovers very slowly. Full recovery is not observed until $\sim 10$ kbar. We note that our results for the PF2/6 film under hydrostatic pressure conditions in the DAC are quite different from that observed by Rothe et al. ${ }^{30}$ where they observe no hysteresis. In their work a film of PF2/6 was sandwiched between two glass slides: the pressure in their case should be substantially lower than that in a DAC, and is uniaxial as well.

The absorption spectrum as a function of pressure was measured from the same film (at the same pressures as the PL) and is shown in the inset of Fig. 4(a). The spectra were taken at room temperature by dividing the sample transmission by the transmission of the empty diamond cell. Such a broad absorption spectrum is characteristic of the $\alpha$ phase. With increasing pressure, the overall absorbance increases and the spectrum redshifts. Increased absorbance in the 2.7 $\mathrm{eV}$ region may be indicative of $\beta$ phase formation. Although the $\alpha$ phase of PF does not show any absorption below 2.90 $\mathrm{eV}$, one cannot rule out a redshift of the absorption edge from the $\alpha$ phase itself with increasing pressures. Such effects have been observed in m-LPPP under pressure. ${ }^{22}$ The cutoff at $2.95 \mathrm{eV}$ is due to the type-II diamond of the pressure cell.

\section{B. Discussion of PL results}

The PL spectrum of the film was fit using six Gaussian peaks. In the inset of Fig. 4(b) we show a spectrum at 14.8 kbar, where the vibronics, keto, and aggregate emissions are all clearly observed. The highest two are the $0-0$ and $0-1$ vibronics. The $2.53 \mathrm{eV}$ peak is close to the $0-2$ position. The 

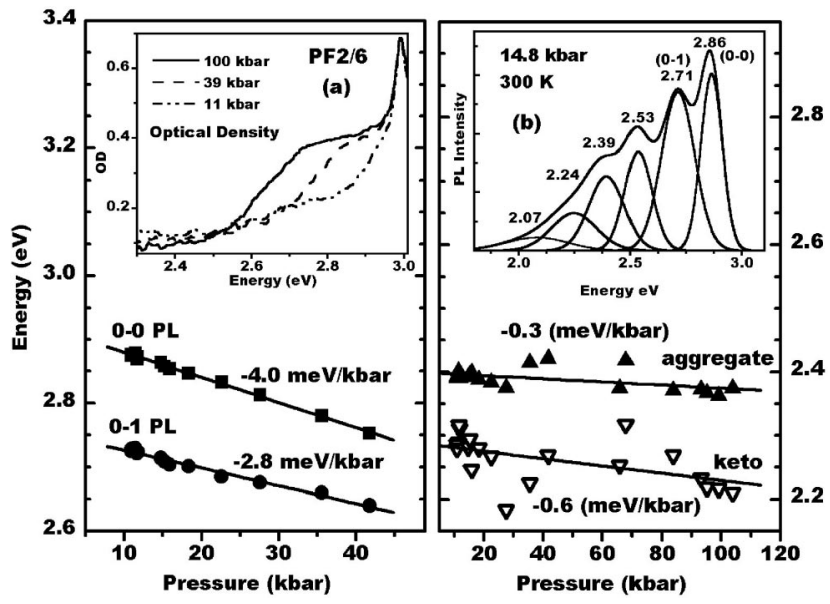

FIG. 4. Peak positions of the various transitions in a film of $\mathrm{PF} 2 / 6$ as a function of increasing pressure at $300 \mathrm{~K}$. The peak position of the $0-0$ and the $0-1$ backbone PL as a function of pressure are shown in (a) and the aggregate and the keto peak positions are shown in (b). The slopes of the various transitions are indicated in the figure. The inset in panel (a) shows the absorption spectra at selected values of pressure. The inset in panel (b) shows the lineshape fits for the 14.8-kbar PL spectrum. The peak positions are in $\mathrm{eV}$.

$2.39 \mathrm{eV}$ peak arises from an aggregate, as does the $2.07 \mathrm{eV}$ peak. The $2.24 \mathrm{eV}$ peak arises from the keto defect. The peak positions of the keto and the aggregate emissions versus pressure for the film sample are plotted in Fig. 4(b), and they redshift by 0.6 and $0.3 \mathrm{meV} / \mathrm{kbar}$, respectively, with increasing pressure. The $0-0$ and the $0-1$ backbone vibronics are clearly visible up to $40 \mathrm{kbar}$ and shift by about an order of magnitude faster [Fig. 4(a)]. Similar systematic fitting of the backbone vibronic peaks is difficult for the powder sample, due to the increasing absorbance and the broad keto emission at higher pressures.

The main impact of pressure on the PL spectrum is the enhanced emission in the 2.1-2.6 eV range which overwhelms the backbone emission of PF2/6 beyond a certain pressure value. Emission in this region has been observed in other works on PF at ambient pressure. Under photodegradation in 9,9-di-n-hexyl-2,7-dibromofluorene (PDHF), three peaks at 2.2, 2.4, and $2.6 \mathrm{eV}$ are observed and have been identified as excimer peaks. ${ }^{10}$ Using time-resolved PL spectroscopy, Herz and Phillips ${ }^{14}$ find a longer decay time for the $2.15 \mathrm{eV}$ peak, also attributed to an excimer type emission, as compared with other peaks in this region. This result has led to a picture that intermolecular interactions dominate the bulk material properties in PFs.

Work by List $e t$ al. ${ }^{12}$ conclusively shows that the 2.2-2.3 $\mathrm{eV}$ emission is from keto defect sites in PF's. This assignment has been confirmed in a recent work by Lupton et al. ${ }^{13}$ using time-resolved PL on a range of poly- and oligofluorene samples. They attribute the $2.3 \mathrm{eV}$ emission to an on-chain emissive defect, in agreement with the keto-type defect. Further, they observe an enhancement of the $2.6 \mathrm{eV}$ emission with increasing sample concentration concluding that the origin of this peak is due to the formation of interchain aggregates.
In our work here, the enhancement of the $2.1-2.6 \mathrm{eV}$ emission for the bulk sample occurs at $\sim 20 \mathrm{kbar}$ and for the film at a slightly higher pressure of $\sim 35 \mathrm{kbar}$. The relative intensities of the various peaks in the $2.1-2.6 \mathrm{eV}$ band under pressure are different in the film and in a powder sample. In bulk PF2/6 (see Fig. 2) both the keto and aggregate emission are equally enhanced at $20 \mathrm{kbar}$ : the keto peak $(\sim 2.3 \mathrm{eV})$ gains intensity over the aggregate peak $(\sim 2.4 \mathrm{eV})$ beyond 42 kbar. Although the concentration of the keto defects is quite small in our PF2/6 sample (as shown in Fig. 1), increasing the intermolecular interaction causes the emission from the defect sites to be considerably enhanced. This scenario is somewhat similar to the guest-host polyfluorene system where a small concentration of a chromophore with an absorption spectrum closely matching the emission of PFO drastically changes the PL emission. ${ }^{31,32}$ In these systems there is an efficient Förster energy transfer to the host molecules that allows conversion of the blue emission from PFO into red emission of the guest chromophore. In our case the enhanced intermolecular interaction allows transfer from the backbone to the keto defect making that emission more and more prominent as pressure increases.

In the PF2/6 film, beyond $35 \mathrm{kbar}$ the $2.1-2.6 \mathrm{eV}$ emission dominates the backbone emission. The aggregate emission is stronger than the keto emission which appears as a weak shoulder at higher pressures. The higher aggregation in the film may result from a more compact and ordered morphology in the film. This ordering may also explain the hysteresis of the PL spectra observed between increasing and decreasing pressures. The absence or weak intensity of ketorelated peaks in the film may result from a "pinning" of excitations in ordered regions that prevents diffusion of the excited carriers to the defect states.

A weak shoulder at $1.9 \mathrm{eV}$ is observed both in the film and powder samples of PF2/6 (see Figs. 2 and 3). The exact origin of this peak is not understood but most probably it is also due to aggregate emission. In Sec. IV we discuss how this PL emission at higher pressures results in an antiresonance effect of the Raman phonons.

The overall PL intensity, due to aggregate and keto emission, increases noticeably at $42 \mathrm{kbar}$ in the powder sample. A temperature scan was taken at this pressure since cooling the sample decreases the pressure by $\sim 2 \mathrm{kbar}$, and thus allows us to cycle through any morphological changes. The relative intensity of the backbone transitions was observed to decrease with increasing temperature. A significant change occurs between 150 and $250 \mathrm{~K}$. Above $250 \mathrm{~K}$ the $0-0$ transition is almost completely quenched and the broadband emission increases in intensity by more than a factor of 3 . These changes in the PL are most likely due to morphological changes in PF2/6. ${ }^{33}$ Transport measurements show that polymers in a liquid crystalline state exhibit greatly enhanced interchain charge-carrier mobility. ${ }^{34}$ Since PF2/6 does exhibit a crystalline to nematic liquid crystalline phase transition, it is possible that such a transition causes the sudden change in the relative intensities of the PL emission with increasing pressures.

The rate of redshift of the 0-0 and 0-1 backbone emission peaks in the PF2/6 film under pressure is similar to shifts 
TABLE I. Pressure coefficients for backbone PL emission peaks in PF2/6 film, m-LPPP film, and PHP powder (beyond $15 \mathrm{kbar}$ ) for the $0-0$ and $0-1$ PL peaks. The pressure coefficients are determined by a linear fit to the PL energy positions vs pressure given by $E(P)=E(0)+\alpha P$.

\begin{tabular}{lcc}
\hline \hline \multicolumn{1}{c}{ Sample } & $\alpha_{0-0}(\mathrm{meV} / \mathrm{kbar})$ & $\alpha_{0-1}(\mathrm{meV} / \mathrm{kbar})$ \\
\hline PF2/6 Film $^{\mathrm{a}}$ & $-4.0 \pm 0.1$ & $-2.8 \pm 0.1$ \\
m-LPPP film $^{\mathrm{b}}$ & $-2.5 \pm 0.1$ & $-2.1 \pm 0.1$ \\
PHP powder $^{\mathrm{b}}$ & $-4.4 \pm 0.2$ & $-5.3 \pm 0.1$ \\
\hline \hline
\end{tabular}

${ }^{\mathrm{a}}$ This work.

${ }^{\mathrm{b}}$ Reference 27.

observed in m-LPPP film and PHP powder, as shown in Table I. Future work on copolymers of PF2/6 and with other side-group substitutions (that minimize the incorporation of keto defects) are required to understand the dynamics of the backbone vibronics in the bulk PF2/6 sample, which barely appear to shift. This effect may well be an artifact due to the higher self-absorption in the powder or due to a different morphology of the powder as compared with the film under hydrostatic pressure. The shifts of the aggregate and keto emissions with pressure are similar in film and powder samples.

\section{RAMAN SCATTERING}

\section{A. Experimental results}

Figure 5 shows the Raman spectra of powder PF2/6 at 13 and $300 \mathrm{~K}$ at ambient pressure. With increasing temperatures all the Raman peaks in the $1200-1600 \mathrm{~cm}^{-1}$ region soften by $\sim 2 \mathrm{~cm}^{-1}$, a value comparable to several known conjugated materials. $^{23}$

The Raman frequencies in the $1050-1200 \mathrm{~cm}^{-1}$ region are sensitive to side group substitution; they arise from the $\mathrm{C}-\mathrm{H}$ bending modes of the ethyl-hexyl side group in PF2/6. The 1290,1342 , and $1417 \mathrm{~cm}^{-1}$ regions are associated with

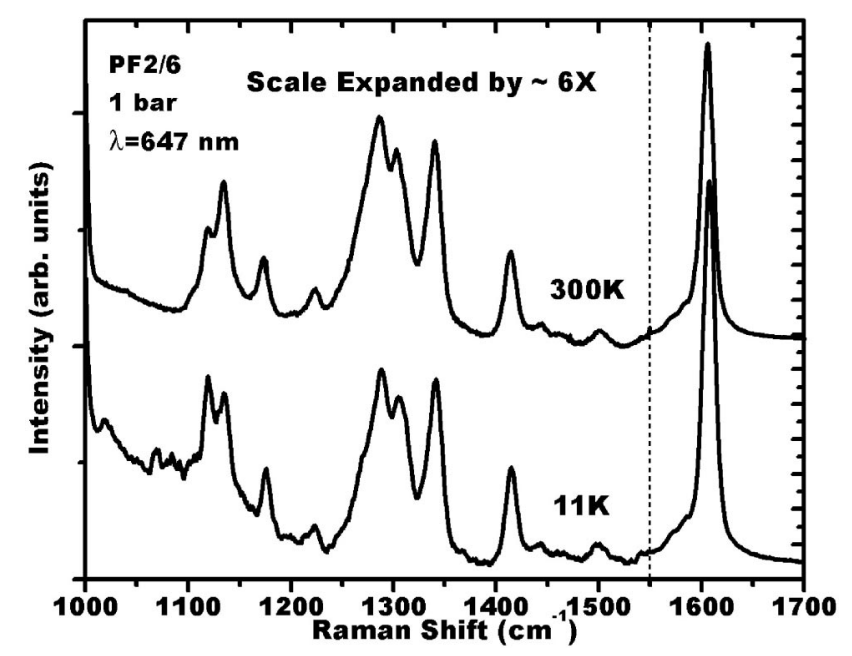

FIG. 5. Raman spectra of PF2/6 at ambient pressure at 13 and $300 \mathrm{~K}$. The vertical scale has been expanded by about $6 \times$ below $1550 \mathrm{~cm}^{-1}$.

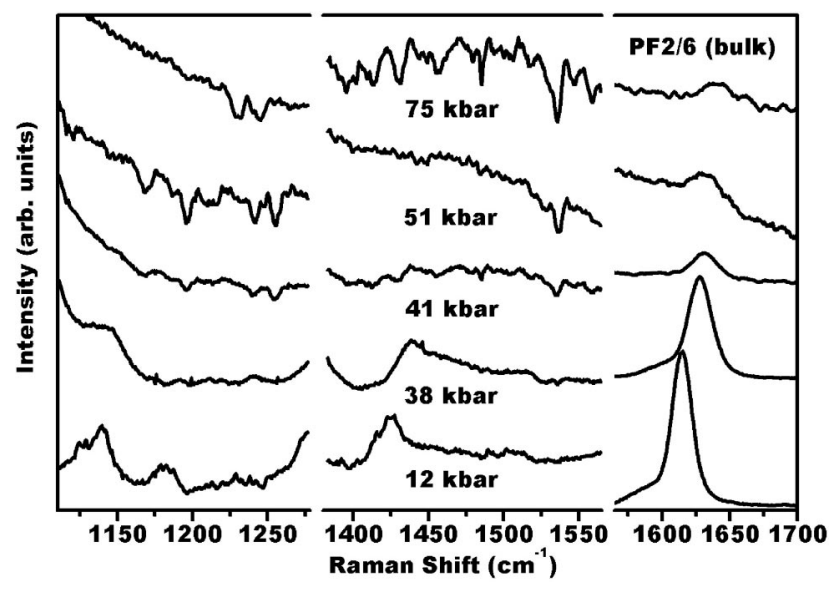

FIG. 6. Raman spectra of PF2/6 at selected pressures. Each frequency region of the graph has been scaled individually to highlight the key features. Owing to the rising PL with increasing pressure, the background increases at higher pressures obscuring the low-frequency Raman peaks.

the backbone $\mathrm{C}-\mathrm{C}$ stretch modes. The Raman peaks in the $1600-\mathrm{cm}^{-1}$ region arise from the intraring $\mathrm{C}-\mathrm{C}$ stretch mode. These peak assignments are similar to those of Ariu et al. ${ }^{35}$ in their work on PFO, where the Raman peaks in the $1200-1400-\mathrm{cm}^{-1}$ region are attributed to the C-C stretch mode between adjacent phenyl rings. It has been assumed that the higher frequency modes in this region are due to the $\mathrm{C}-\mathrm{C}$ stretch from within the more rigid monomer unit, while the $\mathrm{C}-\mathrm{C}$ stretch modes between adjacent monomer units are expected to have a lower frequency due to the allowed torsional degree of freedom. ${ }^{35}$ The $1600-\mathrm{cm}^{-1}$ region of the spectrum is best fit with two peaks, a weak peak at $1582 \mathrm{~cm}^{-1}$ and a strong peak at $1605 \mathrm{~cm}^{-1}$. The weak shoulder at $1580 \mathrm{~cm}^{-1}$ has been observed in other polyfluorenes as well. ${ }^{35,36}$

Figure 6 is a plot of Raman spectra from PF2/6 at various pressures. Each frequency region of the graph has been scaled individually so that key features in each spectrum are clearly observed. The break in the $x$ axis at $1300 \mathrm{~cm}^{-1} \mathrm{de}-$ notes the region where the Raman peak from the diamond has been removed for clarity. Most of the phonon frequencies corresponding to the backbone $\mathrm{C}-\mathrm{C}$ stretch modes are not observable as a function of pressure due to the presence of the strong $1330-\mathrm{cm}^{-1}$ Raman peak from diamonds in the DAC.

As pressure is increased, all of the Raman frequencies analyzed tend to increase in frequency. Concurrently, the PL tail gains intensity, obscuring the Raman signal beginning with the lower frequency peaks. The region below $\sim 1300 \mathrm{~cm}^{-1}$ is observable up to $\sim 20 \mathrm{kbar}$ and the $1417-\mathrm{cm}^{-1}$ peak is observed up to about $40 \mathrm{kbar}$. We note that these Raman measurements were taken using the 647.1-nm $(1.92 \mathrm{eV})$ line of a $\mathrm{Kr}^{+}$laser. Despite being well below the PL peaks of both the backbone and the keto emissions, the laser line still excites the low-energy tail of the PL spectrum (see Fig. 3). We believe that this tail most likely arises from aggregate emissions but possibly could also be due to the keto defects. 
The rising PL tail above 20 kbar causes several of the Raman peaks to exhibit asymmetric line shapes and an antiresonance effect characteristic of a Breit-Wigner Fano (BWF) resonance. Such effects have been observed in inorganic semiconductors, for example in $p$ - and $n$-doped $\mathrm{Si}$ and $\mathrm{Ge}^{37,38}$ This effect is indicative of a strong electron-phonon interaction between the Raman phonons and the electronic continuum. ${ }^{39}$ We find that the most consistent development of the BWF resonance is observed in the $1605-\mathrm{cm}^{-1}$ peak with the development of asymmetry and increased broadening with increasing pressures is seen in Fig. 6. We have conducted a detailed line-shape analysis for this peak in terms of the BWF resonance, discussed in detail in Sec. IV B.

Along with the asymmetry of the $1605-\mathrm{cm}^{-1}$ peak, new peaks exhibiting antiresonance effects appear near 1230 and $1530 \mathrm{~cm}^{-1}$ (as seen in Fig. 6). These peaks appear in the Raman spectra both with increasing and decreasing pressure. Although their signals are weak, their spectra are reproducible. Some of them may be infrared (IR) active modes that become Raman active at higher pressures. In Ref. 12 it is seen that there are a number of IR peaks in the $1000-1500-\mathrm{cm}^{-1}$ range in PF with the strongest IR peak appearing at $1500 \mathrm{~cm}^{-1}$. The $1530 \mathrm{~cm}^{-1}$ feature observed around $40 \mathrm{kbar}$ is most likely the $1500 \mathrm{~cm}^{-1}$ IR active peak shifted to higher frequencies due to the applied pressure. This IR frequency shows a weak signature even in the 1 bar Raman spectrum (Figs. 5 and 6), and the magnitude of the shift is consistent with the pressure shifts of the other Raman peaks, as seen in Fig. 7.

\section{B. Discussion of Raman results}

The Raman peaks have been fit with Lorentzian line shapes to determine their frequency positions. Their peak positions as a function of pressure are plotted in Fig. 7. The $1417 \mathrm{~cm}^{-1}$ mode is seen clearly up to $40 \mathrm{kbar}$. The $1500 \mathrm{~cm}^{-1}$ peak is too weak in intensity to track below 40 kbar, but can be seen as a marked antiresonance and can be tracked reliably for a few data points above $40 \mathrm{kbar}$. The 34-kbar data point for this peak was taken as the pressure was being decreased, and due to a hysteresis effect the peak was still visible. As the pressure was decreased further the PL background dropped in intensity and the low pressure spectra were fully recovered. All of the peaks shift with fairly similar pressure coefficients.

The positions of the $1605 \mathrm{~cm}^{-1}$ and the shoulder peak at $1582 \mathrm{~cm}^{-1}$ are included up to $60 \mathrm{kbar}$ in Fig. 7. Above about $40 \mathrm{kbar}$ the increase of the asymmetry of the $1605 \mathrm{~cm}^{-1}$ peak made fitting to the Lorentzian line shape difficult. Since the interaction of the main $1605 \mathrm{~cm}^{-1}$ peak with the electronic continuum makes the line shape broader and asymmetric it was more accurately determined by a BWF fit. It is possible only to resolve one peak using a BWF line shape. We have fit the entire pressure range using both fits (see Fig. 7). At lower pressures there is good agreement between the peak frequencies determined by both the Lorentzian and the BWF fits, however, the values deviate at higher pressures.

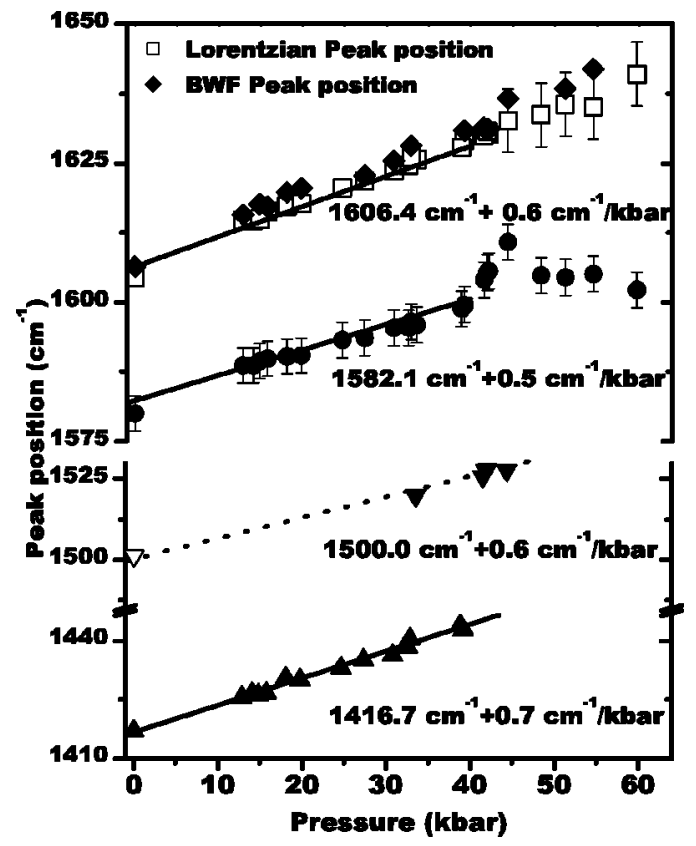

FIG. 7. Peak positions of the 1417-, 1500- (IR-active), 1582(shoulder of $1605 \mathrm{~cm}^{-1}$ ), and $1605 \mathrm{~cm}^{-1}$ modes as a function of pressure. The open symbol $(\nabla)$ denotes the position of the $1500-\mathrm{cm}^{-1}$ peak at 1 bar. The slopes for the linear fits have an uncertainty of $\pm 0.05 \mathrm{~cm}^{-1} / \mathrm{kbar}$. The straight lines for the 1605 and the $1582-\mathrm{cm}^{-1}$ peaks are fits to the frequencies obtained from the Lorentzian fits up to $40 \mathrm{kbar}$.

Our linear fits to the frequency vs pressure data for the Lorentzian fits are shown in Fig. 7 up to 40 kbar.

Since the PL emission from the aggregates and the keto defects redshift, at higher pressures the 647.1-nm Raman excitation line $(1.92 \mathrm{eV})$ excites the tail of the emission (Fig. 2 ), which couples strongly to the phonons. We therefore directly excite the tail of the emission, which is most likely from defect and aggregate emission, without exciting the backbone PL emission. This excludes the possibility of this low-energy emission being from excimers.

To determine the peak position, asymmetry parameter and linewidth as a function of pressure, we fit the $1605 \mathrm{~cm}^{-1}$ peak with a BWF line shape given by ${ }^{40}$

$$
I(\omega)=I_{0} \frac{\left[\left(\omega-\omega_{0}\right) / q+\Gamma\right]^{2}}{\left(\omega-\omega_{0}\right)^{2}+\Gamma^{2}},
$$

where $\omega_{0}$ is the discrete phonon frequency and $\Gamma$ is the width of the resonant interference between the continuum and discrete scattering channels. The asymmetry parameter $(1 / q)$ depends on the average electron-phonon matrix element $M$ and the Raman matrix elements between the ground and excited states of the phonon and electron. ${ }^{37}$ The broadening parameter is given by

$$
\Gamma=\pi M^{2} D(\omega),
$$

where $D(\omega)$ is the combined density of states for the electronic transitions. For nonzero density of states it turns out 


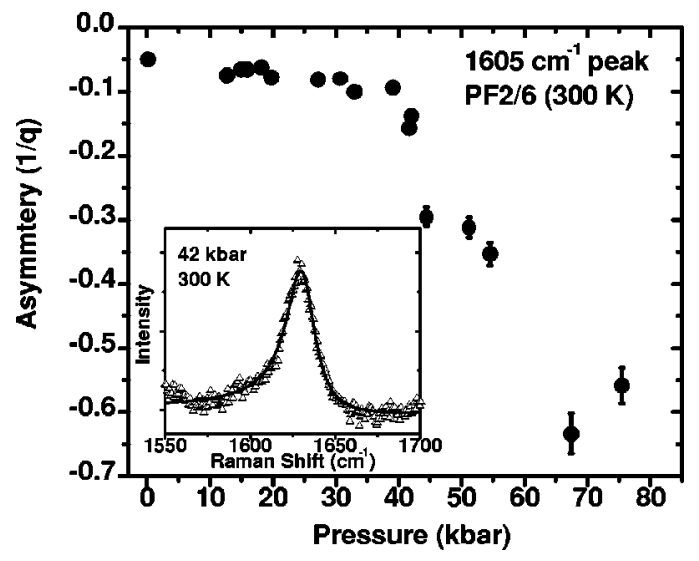

FIG. 8. Asymmetry parameter $(1 / q)$ of the $1605 \mathrm{~cm}^{-1}$ Raman peak versus pressure. $1 / q$ is obtained by fits to the Raman peak with a BWF line shape [Eq. (1)]. The inset shows a sample BWF fit to the $1605 \mathrm{~cm}^{-1}$ peak at $42 \mathrm{kbar}$.

that when $q=-\left(\omega-\omega_{0}\right) / \Gamma$, the spectral function $I(\omega)$ will reveal an "antiresonance" close to the value of phonon frequency. 39

Figure 8 shows the asymmetry parameter versus pressure for the $1605 \mathrm{~cm}^{-1}$ Raman mode. The asymmetry is relatively small up to about $35 \mathrm{kbar}$, with a $1 / q$ value between -0.005 and 0.1 . In this lower pressure region the Lorentzian and BWF fits give very similar results. Beyond $40 \mathrm{kbar}$, however, the asymmetry increases rapidly and $1 / q$ varies from -0.3 to -0.6 . The inset shows a sample BWF fit to Eq. (1) (bold line) to the experimental data (open triangles) for data at $42 \mathrm{kbar}$. The peak position and the linewidths from the BWF fits as a function of pressure are plotted in Fig. 9. The linewidth shows a square law dependence with

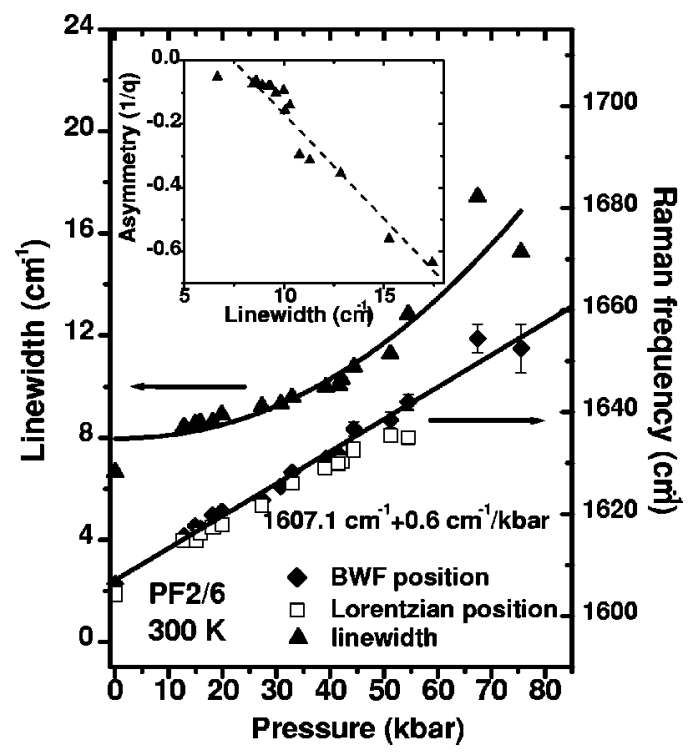

FIG. 9. Linewidth and the peak position of the $1605 \mathrm{~cm}^{-1} \mathrm{Ra}-$ man peak obtained by fits to Eq. (1), as a function of pressure. A linear fit to the BWF peak position of the $1605 \mathrm{~cm}^{-1}$ Raman peak as a function of pressure yields a slope of $0.6 \pm 0.02 \mathrm{~cm}^{-1} / \mathrm{kbar}$, as shown by the straight line. The inset shows the asymmetry parameter to linearly vary with the linewidth. pressure $\left(\Gamma \propto P^{2}\right)$ (solid line in Fig. 9). The frequency positions vary almost linearly with pressure. The inset of Fig. 9 shows $1 / q$ to linearly vary with the linewidth $\Gamma$.

Recently, optical techniques based on photoinduced infrared-active vibrational (IRAV) modes have been used to study the photogeneration and recombination dynamics of charged polarons in conjugated polymers. ${ }^{41}$ Österbacka et al. have observed Fano-type antiresonances in the IRAV modes in a series of $\pi$-conjugated polymers that are explained well by extending the amplitude model beyond the adiabatic limit. ${ }^{42}$ Our observation here is somewhat different; the electronic continuum is from a combination of aggregate and defect emissions that shift to lower energies with increasing pressure. Figure 8 shows that $q$ is negative, indicating that the center frequency of the continuum lies below the discrete mode frequency of $1605 \mathrm{~cm}^{-1}(0.2 \mathrm{eV})$. It is interesting to point out that in $M_{3} C_{60}$ and superconducting cuprates, $1 / q$ value lies between -0.2 and $-0.5,{ }^{40}$ similar to our results beyond $40 \mathrm{kbar}$. The high background and the interference from the diamond Raman peak makes it difficult to analyze the asymmetry of the other Raman peaks as a function of pressure. The systematic appearance of the inverted $1530 \mathrm{~cm}^{-1}$ peak at higher pressures makes it a viable candidate for the IR-active frequency that gets activated in the Raman spectrum due to a lowering of the symmetry. Most probably the peak shows an $s$-like behavior due to the antiresonance effect and therefore appears as a negative peak. We have cycled the pressure many times to confirm the antiresonance effect on the $1530 \mathrm{~cm}^{-1}$ peak: the $s$-like behavior disappears at lower pressures but appears consistently at higher pressure values.

Since the vibrational frequencies of a harmonic solid are independent of compression, pressure induced changes in the Raman spectrum provide insight into the anharmonicity of the solid-state potential. ${ }^{43}$ In this light the linear shift of the $1605 \mathrm{~cm}^{-1}$ peak as a function of pressure is not surprising. The rate of shift is similar to other Raman-active frequencies in PF2/6 which is an indication of the anharmonic potential. Although one expects an additional shift in phonon frequency due to the BWF interaction (the real part of the selfenergy) we cannot infer any information about this parameter from our data. In materials where the BWF interaction can be tuned, e.g., using uniaxial stress in both $n$-type ${ }^{37}$ and $p$-type $\mathrm{Si}^{38}$ the real part of the self-energy due to interaction between the phonon and the electronic continuum is determined by comparison with the pure material. In this work, such a comparison is not possible owing to a lack of a defect-free but identical material. Had this parameter been large one would expect a significant deviation in the peak position vs pressure behavior above $40 \mathrm{kbar}$ where the BWF character of the mode is pronounced.

However, the imaginary part of the self-energy, $\Gamma$, does show a quadratic dependence with pressure. Furthermore, the asymmetry parameter should be directly proportional to $\Gamma$ if the average electron-phonon matrix element and the Raman matrix elements between the ground and excited states are assumed to be roughly constant. ${ }^{44}$ Indeed, this is what we find, as shown in the inset of Fig. 9. The negative slope here is due to the negative $q$ values that we obtain from the fit. At 
present we are unclear of the physical significance of the quadratic dependence of $\Gamma$ on pressure, which reflects on the density of states of the continuum. A full-scale bandstructure calculation incorporating aggregate and defect states should reveal the nature of the electronic density of states.

\section{CONCLUSION}

In conclusion, we have shown that hydrostatic pressure induces very efficient energy transfer of the singlet excitons to the keto defect sites mainly in bulk PF2/6. Enhanced intermolecular interaction induces a strong aggregate-type emission in the film sample, in addition to a keto emission. This may result from a pinning of excitations in ordered regions in the film that prevents excitation diffusion to the defect states. The Raman modes harden with increasing pressures. The $1605 \mathrm{~cm}^{-1}$ peak exhibits an asymmetric line shape, characteristic of a Breit-Wigner Fano resonance, and some of the other peaks show an antiresonance effect. This is indicative of a strong electron-phonon interaction between the Raman phonons and the electronic continuum. We have carried out a detailed BWF line-shape analysis for the $1605 \mathrm{~cm}^{-1}$ peak as a function of pressure.

The asymmetry observed in the Raman lines is fully consistent with the PL data, i.e., the BWF resonance is pronounced beyond $40 \mathrm{kbar}$ where the PL emission drastically changes. The aggregate and defect related emission increases significantly and the asymmetry of the $1605 \mathrm{~cm}^{-1}$ Raman peak becomes higher. Future work on different side-group substituted PFs that have extraordinarily small keto defect concentration are in progress to obtain insight into the morphological changes that occur in PFs as a function of hydrostatic pressure.

\section{ACKNOWLEDGMENT}

S.G. acknowledges the donors of the American Chemical Society Petroleum Research Fund (No. 38193-B7) for partial support of this research. U.S. thanks SONY International Europe, Stuttgart, and the Deutsche Forschungsgemeinschaft (DFG) for financial support. One of us (S.G.) thanks Michael Winokur and Carsten Ullrich for many valuable insights and discussions.
*Corresponding author. Present address: Department of Physics, University of Missouri, Columbia, MO 65211, USA. Email address: guhas@missouri.edu

${ }^{1}$ M. Leclerc, J. Polym. Sci., Part A: Polym. Chem. 39, 2867 (2001)

${ }^{2}$ U. Scherf and E.J.W. List, Adv. Mater. (Weinheim, Ger.) 14, 477 (2002).

${ }^{3}$ A.W. Grice, D.D.C. Bradley, M.T. Bernius, M. Inbasekaran, W.W. Wu, and E.P. Woo, Appl. Phys. Lett. 73, 629 (1998).

${ }^{4}$ M. Redecker, D.D.C. Bradley, M. Inbasekaran, and E.P. Woo, Appl. Phys. Lett. 73, 1565 (1998).

${ }^{5}$ S. Tasch, E.J.W. List, C. Hochfilzer, G. Leising, P. Schlichting, U. Rohr, Y. Geerts, U. Scherf, and K. Müllen, Phys. Rev. B 56, 4479 (1997).

${ }^{6}$ M.T. Bernius, M. Inbasekaran, J. O’Brien, and W. Wu, Adv. Mater. (Weinheim, Ger.) 12, 1737 (2000).

${ }^{7}$ T. Miteva, A. Meisel, M. Grell, H.G. Nothofer, D. Lupo, A. Yasuda, W. Knoll, L. Kloppenburg, U.H.F. Bunz, U. Scherf, and D. Neher, Synth. Met. 111, 173 (2000).

${ }^{8}$ M. Grell, D.D.C. Bradley, G. Ungar, J. Hill, and K.S. Whitehead, Macromolecules 32, 5810 (1999).

${ }^{9}$ A.J. Cadby, P.A. Lane, H. Mellor, S.J. Martin, M. Grell, C. Giebeler, D.D.C. Bradley, M. Wohlgenannt, C. An, and Z.V. Vardeny, Phys. Rev. B 62, 15604 (2000).

${ }^{10}$ V.N. Bliznyuk, S.A. Carter, J.C. Scott, K. Klrner, R.D. Miller, and D.C. Miller, Macromolecules 32, 361 (1999).

${ }^{11}$ K.H. Weinfurtner, H. Fujikawa, S. Tokito, and Y. Taga, Appl. Phys. Lett. 76, 2502 (2000).

${ }^{12}$ E.J.W. List, R. Guentner, P.S. de Freitas, and U. Scherf, Adv. Mater. (Weinheim, Ger.) 14, 374 (2002).

${ }^{13}$ J.M. Lupton, M.R. Craig, and E.W. Meijer, Appl. Phys. Lett. 80, 4489 (2002).

${ }^{14}$ L.M. Herz and R.T. Phillips, Phys. Rev. B 61, 13691 (2000).

${ }^{15}$ S. Tasch, A. Niko, G. Leising, and U. Scherf, Appl. Phys. Lett. 68, 1090 (1996).
${ }^{16}$ Y. Yang, Q. Pei, and A.J. Heeger, Appl. Phys. Lett. 79, 934 (1996).

${ }^{17}$ D. Beljonne, Z. Shuai, R.H. Friend, and J.L. Bredas, J. Chem. Phys. 102, 2022 (1995).

${ }^{18}$ J. Cornil, A.J. Heeger, and J.L. Bredas, Chem. Phys. Lett. 272, 463 (1997).

${ }^{19}$ F.C. Spano, J. Chem. Phys. 114, 5376 (2001).

${ }^{20}$ P. Puschnig and C. Ambrosch-Draxl, Phys. Rev. Lett. 89, 056405 (2002).

${ }^{21}$ S. Yang, W. Graupner, S. Guha, P. Puschnig, C. Martin, H.R. Chandrasekhar, M. Chandrasekhar, G. Leising, C. AmbroschDraxl, and U. Scherf, Phys. Rev. Lett. 85, 2388 (2000).

${ }^{22}$ M. Chandrasekhar, S. Guha, and W. Graupner, Adv. Mater. (Weinheim, Ger.) 13, 613 (2001).

${ }^{23}$ S. Guha, W. Graupner, R. Resel, M. Chandrasekhar, H.R. Chandrasekhar, R. Glaser, and G. Leising, Phys. Rev. Lett. 82, 3625 (1999); J. Phys. Chem. A 105, 6203 (2001).

${ }^{24}$ G. Lieser, M. Oda, T. Miteva, A. Meisel, H.-G. Nothofer, U. Scherf, and D. Neher, Macromolecules 33, 4490 (2000).

${ }^{25}$ D. Moses, A. Feldblum, E. Ehrenfreund, A.J. Heeger, T.-C. Chung, and A.G. MacDiarmid, Phys. Rev. B 26, 3361 (1982).

${ }^{26}$ B.C. Hess, G.S. Kanner, and Z.V. Vardeny, Phys. Rev. B 47, 1407 (1993).

${ }^{27}$ S. Guha, S. Yang, W. Graupner, M. Chandrasekhar, H.R. Chandrasekhar, and G. Leising, Phys. Status Solidi B 211, 177 (1999).

${ }^{28}$ S. Webster and D.N. Batchelder, Polymer 37, 4961 (1996).

${ }^{29}$ G. Yang, Y. Li, J.O. White, and H.G. Drickamer, J. Phys. Chem. B 103, 7853 (1999).

${ }^{30}$ C. Rothe, S.I. Hintschich, L.-O. Palsson, A.P. Monkman, R. Guentner, and U. Scherf, Chem. Phys. Lett. 360, 111 (2002).

${ }^{31}$ T. Virgili, D.G. Lidzey, and D.D.C. Bradley, Adv. Mater. (Weinheim, Ger.) 12, 58 (2000).

${ }^{32}$ G. Cerullo, S. Stagira, M. Z-Rossi, S. DeSilverstri, T. Virgili, 
D.G. Lidzey, and D.D.C. Bradley, Chem. Phys. Lett. 335, 27 (2001).

${ }^{33} \mathrm{X}$-ray studies of fluorene under high pressure show crystallographic phase changes starting at about $40 \mathrm{kbar}$, M. Winokur (private communication).

${ }^{34}$ M. Redecker, D.D.C. Bradley, M. Inbasekaran, and E.P. Woo, Appl. Phys. Lett. 74, 1400 (1999).

${ }^{35}$ M. Ariu, D.G. Lidzey, and D.D.C. Bradley, Synth. Met. 111-112, 607 (2000).

${ }^{36}$ H. Leim, P. Etchegoin, K.S. Whitehead, and D.D.C. Bradley, J. Appl. Phys. 92, 1154 (2002).

${ }^{37}$ M. Chandrasekhar, J.B. Renucci, and M. Cardona, Phys. Rev. B 17, 1623 (1978).

${ }^{38}$ L. Pintschovius, J.A. Verges, and M. Cardona, Phys. Rev. B 26,
5658 (1982).

${ }^{39}$ M.V. Klein, Light Scattering in Solids, edited by M. Cardona (Springer-Verlag, New York, 1975).

${ }^{40}$ P. Zhou, K-A. Wang, P.C. Eklund, G. Dresselhaus, and M.S. Dresselhaus, Phys. Rev. B 48, 8412 (1993).

${ }^{41}$ P.B. Miranda, D. Moses, and A.J. Heeger, Phys. Rev. B 64, 081201 (2001).

${ }^{42}$ R. Österbacka, X.M. Jiang, C.P. An, B. Horovitz, and Z.V. Vardeny, Phys. Rev. Lett. 88, 226401 (2002).

${ }^{43}$ J.R. Ferraro, Vibrational Spectroscopy at High External Pressures (Academic Press, New York, 1984).

${ }^{44}$ S. Satpathy, M. Chandrasekhar, H.R. Chandrasekhar, and U. Venkateswaran, Phys. Rev. B 44, 11339 (1991). 